\title{
LOCALIZATION SCHEMES FOR UNDERWATER WIRELESS SENSOR NETWORKS: SURVEY
}

\author{
Faiza Al-Salti ${ }^{1}$, N. Alzeidi ${ }^{2}$ and Khaled Day ${ }^{2}$ \\ ${ }^{1}$ Department of Computing, Muscat College, Oman \\ ${ }^{2}$ Department of Computer Science, Sultan Qaboos University, Oman
}

\begin{abstract}
Underwater Wireless Sensor Networks (UWSNs) enable a variety of applications such as fish farming and water quality monitoring. One of the critical tasks in such networks is localization. Location information can be used in sensor networks for several purposes such as (i) data tagging in which sensed information is not useful for the application unless the location of the sensed information is known, (ii) tracking objects or (iii) multi-hop data transmission in geographic routing protocols. Since GPS does not work well underwater, several localization schemes have been developed for UWSNs. This paper surveys the state-ofthe-art of localization schemes for UWSNs. It describes the existing schemes and classifies them into different categories. Furthermore, the paper discusses some open research issues that need further investigation in this area.
\end{abstract}

\section{KEYWORDS}

Underwater Wireless Sensor Networks, Localization, Ranging Methods, Positioning, Range-based \&Range-free.

\section{INTRODUCTION}

With the increasing importance of oceans and seas, there is a strong demand to investigate the unexplored regions and make use of their valuable treasures. Underwater Wireless Sensor Networks (UWSNs) are considered a promising candidate for exploring the oceans and enabling various applications such as fish farming and military surveillance. However, the nature of the underwater environment and the characteristics of the underwater communication media impose several challenges for the design of UWSNs.

Localization is considered as one of the critical tasks, and it can be defined as the process of finding out the location of an object in a given coordinate system. It can be determined using rectangular coordinates $(\mathrm{x}, \mathrm{y}, \mathrm{z})$, cylindrical coordinates $(\mathrm{p}, \varphi, \mathrm{z})$ or spherical coordinates $(\mathrm{r}, \varphi, \theta)$ as demonstrated in Figure 1 for the object labelled as S. In sensor networks, location information can be used for different purposes. For instance, it can be used for data tagging in which sensed information is not useful for the application unless the location of the sensed information is known. Moreover, it can be used for tracking objects or for multi-hop data transmission (geographic routing protocols are based on sensor nodes' locations). Localization schemes developed for UWSNs assume that there are special devices with known locations upon which the localization algorithm is built [1]. 


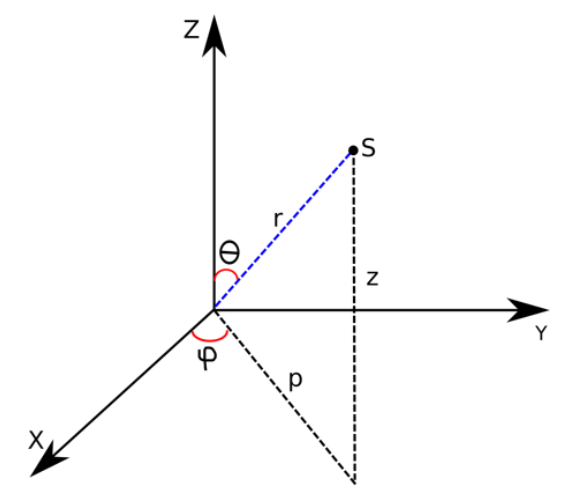

Figure 1: Illustration of the three coordinate systems

Generally, localization schemes aim to achieve the following objectives [2][3]:

- Large coverage: By ensuring that most of the sensor nodes are able to obtain their location information.

- Low communication overhead: Location information of the sensor nodes should be estimated using a small number of packets to save network's energy and reduce channel congestion.

- High accuracy: A localization algorithm should reduce the error in the estimated location information by reducing the gap between the estimated location and the true location.

- Low deployment cost: The deployment of surface buoys and anchor nodes (defined below) or any other special devices used to help in locating nodes should be feasible and cost-effective.

- Good scalability: The complexity of the localization algorithm should grow efficiently (for example linearly) as a function of the number of nodes.

The rest of this paper is organized as follows. Section 2 introduces and describes some of the terms and concepts used throughout the paper. This is followed in Section 3 by a classification of localization schemes into different categories. Section 4 describes the existing localization schemes that are developed for UWSNs. Some open research issues related to localization in UWSNs are discussed in Section 5. Section 6 concludes the paper.

\section{Preliminaries}

This section introduces some of the terms and concepts used throughout the paper.

\subsection{Network Devices}

When discussing localization in UWSNs, there is a need to differentiate between different types of devices (nodes).

- Surface buoys: Nodes with known locations (probably equipped with GPS receivers) and deployed on the water surface. 
International Journal of Computer Networks \& Communications (IJCNC) Vol.12, No.3, May 2020

- Underwater anchor nodes: Nodes with known locations deployed underwater. Yet, anchors and buoys are used synonymously in some published articles. Surface buoys and anchor nodes are used to locate nodes with unknown locations.

- Ordinary nodes: Nodes deployed underwater that need to be located.

- Reference nodes: A reference node is a node with a known location. It could be a surface buoy, an anchor node or an ordinary node after being located. These nodes help in locating nodes with unknown locations.

\subsection{Ranging Methods}

Several ranging methods are used to estimate the distance between two communicating nodes. The following is a description of these methods:

- Time of Arrival (ToA): ToA is the propagation time required for a signal to be received by the receiver. It is used to estimate the distance between the two communicating nodes by multiplying it with the speed of the signal (acoustic speed in the case of UWSNs). However, this method requires clock synchronization. Since clock synchronization is a challenging task in UWSNs, some of the localization schemes use request/reply packet exchanges to calculate the ToA (i.e. called two-way ranging method) [1]. Although it eliminates the need for synchronization, it might incur communication overhead and drains nodes' energy. Also, it might result inan inaccurate estimation of the propagation time due to the asymmetric property of the acoustic channels in underwater. Despite all of these, ToA is considered as the best ranging method to be used in underwater localization [1].

- Time Difference of Arrival (TDoA): This is based on measuring the difference in the arrival time of two signals (e.g. Radio Frequency (RF) and acoustic signals) [1]. Since RF is not propagated well in underwater environment, this method measures the time difference in the arrival between two packets received from two different reference nodes [3].

- Angle of Arrival (AoA): AoA measures the angle between the signal's propagation path and a predefined reference direction. Because of the expensive directional antennas and their sizes, AoA is rarely used in UWSNs [1].

- Received Signal Strength Indicator (RSSI): RSSI estimates the distance between the sender and the receiver by measuring the propagation loss of the signal. In other words, this method measures the difference between the transmitted power and the received power of the signal and compares it with a propagation loss model [1]. RSSI incurs less overhead [4]; however, it is not preferable in UWSNs because the propagation loss of an underwater acoustic signal is temporally-variable. Also, an acoustic signal is affected by multipath and fading effects, which result in inaccurate distance estimation [3].

\subsection{Lateration}

Lateration is a technique used to estimate the location coordinates of an object (the target) given a number of objects (anchors) with known locations. Anchors are in the communication range of the target. The method is well-known and it is also used by GPS [1][5]. Lateration is based on the idea of intersecting circles in which anchors are centered in the circles as shown in Figure 2. The location of the target object in the $(x, y)$ coordinates is in the intersecting area (shading area) of the three circles. In other words, we need to solve a set of equations of the form: 
International Journal of Computer Networks \& Communications (IJCNC) Vol.12, No.3, May 2020

$$
\left(x_{1}-x\right)^{2}+\left(y_{1}-y\right)^{2}=d_{1}^{2}(1)
$$

Where $\left(x_{l}, y_{1}\right)$ is the location of an anchor, $(x, y)$ is the location of the target, and $d_{l}$ is the distance between the anchor and the target. Note that the distance can be determined a priori using one of the ranging methods (e.g. ToA, TDoA) described above.

To further demonstrate the idea, let us use the example of Figure 2. Assume that the three anchors have the locations $\left(x_{i}, y_{i}\right)$ where $i=\{1,2,3\}$ and the target $S$ has the location $(x, y)$. The set of equations is as shown in (2).

$$
\left[\begin{array}{l}
\left(x_{1}-x\right)^{2}+\left(y_{1}-y\right)^{2} \\
\left(x_{2}-x\right)^{2}+\left(y_{2}-y\right)^{2} \\
\left(x_{3}-x\right)^{2}+\left(y_{3}-y\right)^{2}
\end{array}\right]=\left[\begin{array}{l}
d_{1}{ }^{2} \\
d_{2}{ }^{2} \\
d_{3}{ }^{2}
\end{array}\right]
$$

Subtracting the third equation from the first two equations yields the following system of equations $A X=b$ :

$$
\left[\begin{array}{l}
2\left(x_{1}-x_{3}\right)+2\left(y_{1}-y_{3}\right) \\
2\left(x_{2}-x_{3}\right)+2\left(y_{2}-y_{3}\right)
\end{array}\right]\left[\begin{array}{l}
x \\
y
\end{array}\right]=\left[\begin{array}{l}
x_{1}{ }^{2}+y_{1}{ }^{2}-x_{3}{ }^{2}-y_{3}{ }^{2}+d_{3}{ }^{2}-d_{1}{ }^{2} \\
x_{2}{ }^{2}+y_{2}{ }^{2}-x_{3}{ }^{2}-y_{3}{ }^{2}+d_{3}{ }^{2}-d_{2}{ }^{2}
\end{array}\right]
$$

, where

$$
\begin{gathered}
A=\left[\begin{array}{l}
2\left(x_{1}-x_{3}\right)+2\left(y_{1}-y_{3}\right) \\
2\left(x_{2}-x_{3}\right)+2\left(y_{2}-y_{3}\right)
\end{array}\right], X=\left[\begin{array}{l}
x \\
y
\end{array}\right], \text { and } \\
b=\left[\begin{array}{l}
x_{1}{ }^{2}+y_{1}{ }^{2}-x_{3}{ }^{2}-y_{3}{ }^{2}+d_{3}{ }^{2}-d_{1}{ }^{2} \\
x_{2}{ }^{2}+y_{2}{ }^{2}-x_{3}{ }^{2}-y_{3}{ }^{2}+d_{3}{ }^{2}-d_{2}{ }^{2}
\end{array}\right]
\end{gathered}
$$

The matrix $X$ can be solved using the equation given in (4) [6][5]:

$$
X=\left(A^{T} A\right)^{-1} A^{T} b \quad(4)
$$

Generally, to determine a location in $n$ coordinates; at least $n+l$ anchors are needed. In UWSNs, for example, we need four anchors to determine the location in three dimensions $(x, y, z)$. The equation looks like (5):

$$
\left(x_{i}-x\right)^{2}+\left(y_{i}-y\right)^{2}+\left(z_{i}-z\right)^{2}=d_{i}^{2}
$$

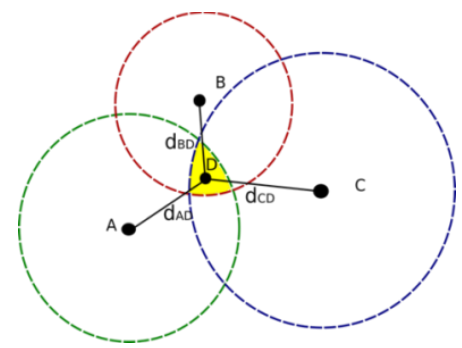

Figure 1:Illustration of the lateration method 


\subsection{Angulation}

While lateration uses distances to estimate the position of the node, angulation is based on angles determined from AoA measurement. It then exploits the geometric principles of triangles to estimate the position. For estimating a location in a $2 \mathrm{D}$ space, there is a need for at least two reference nodes. In a 3D space, the azimuth measure is also needed. To explain the method further, let us consider Figure 3. Assume that $A$ and $B$ are two reference nodes, $S$ is the to-belocated node. The angles $\phi 1, \phi 2$, and the distance $\overline{\overline{A B}}$ are known to $S$. Then, from the trigonometry laws of sines and cosines, $S$ can be located at the distance $\overline{\overline{S C}}$ perpendicular to $\overline{\overline{A B}}$. $\overline{\overline{S C}}$ can be calculated as given in (6).

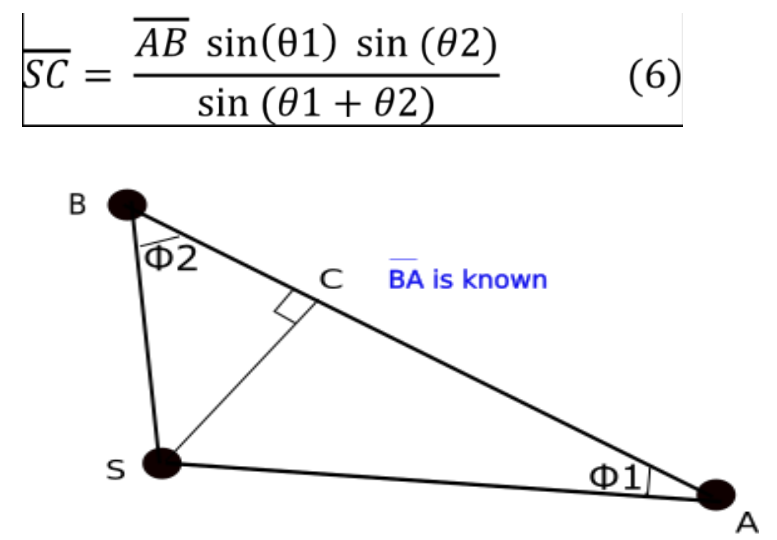

Figure 2: Illustration of the triangulation method

\subsection{Bounding Box}

The bounding box is another method used to estimate a node's location. While lateration relies on the intersection of circles, the bounding box is based on the intersection of rectangles. The location and distance of at least two reference nodes are needed. To explain the method further, consider the example in Figure 4. Let $A$ and $B$ be the reference nodes and $S$ the ordinary node to be located. $S$ is assumed to know the locations of $A$ and $B$. Also, it is assumed to know the distances to these reference nodes (it can be estimated using any of the distance measurements explained earlier). Then, $S$ draws a bounding box for each reference node based on its location and the transmission range (say $R$ ) as follows. The location of the reference node is assumed the center of the box and the length of the side is $2 R$. The center of the intersected rectangles (labelled as $\overline{S^{\prime}}$ ) is considered as the estimated location of S. Although, the bounding box requires less computation cost, it can just provide a course-grained estimation [7].

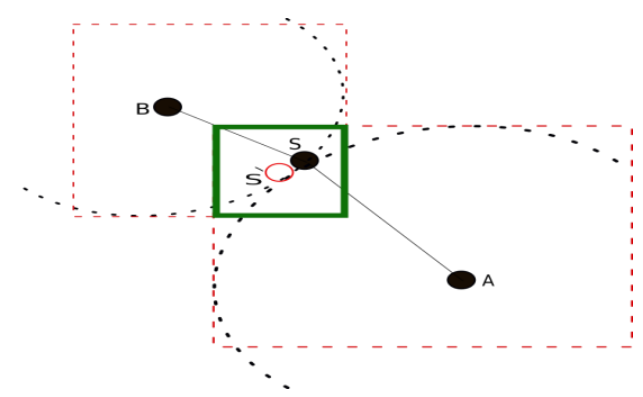

Figure 3: Illustration of the bounding box 


\subsection{Projection}

UWSNs are characterized by their 3D environment; hence, sensor nodes need to be located in a 3D space. This requires that at least one reference node among those used to locate an ordinary node is located underwater. However, it is a challenging task to locate anchors underwater (i.e. if there is a way to locate underwater anchors, then, ordinary nodes can be located in the same way)[8]. Since depth information can be easily determined using pressure sensors equipped in the sensor nodes, the $3 \mathrm{D}$ localization problem can be reduced to a $2 \mathrm{D}$ problem via a projection technique. This is done by projecting the location of the reference nodes into the horizontal plane of the to-be located ordinary node as shown in Figure 5. Nevertheless, this projection needs to be non-degenerative (i.e. no two reference nodes have the same xy-coordinates) [8][9]. It is worth mentioning that the distance to be used after projection is not the original distance $d$. Instead, the ordinary node computes the distance $\overline{d^{\prime}}$ to a projected reference node $i$ as in (7), where $z_{i}$ is the depth of the reference node, and $z$ is the depth of the ordinary node.



Figure 4: Demonstration of the projection technique

\section{Classifications of LOCALIZATION SCHEMES}

Localization schemes for UWSNscan be classified based on different criteria as follows:

- Range measurement: localization schemes can be classified based on range measurement into range-based, range-free and hybrid. Range-based schemes usea range or bearing information, which can be calculated using techniques such as ToA, TDoA, AoA or RSSI. Then, the nodes' locations can be estimated via lateration or angulation methods. Schemes in this category provide a fine-grained estimation of the location. On the other hand, range-free localization schemes do not depend on range or bearing information. Instead, they utilize topology and connectivity of the nodes to estimate nodes' locations [10]. Distance Vector-hop (DV-hop) [11] and Approximate Point In Triangle (APIT) [12] are examples of methods used to estimate locations in range-free schemes. Although range-free schemes are simple, they provide only coarse-grained location estimation. Animal tracking is one of the applications that might require coarse-grained localization [13]. Finally, hybrid schemes combine both range-based and range-free methods to determine location coordinates. For example, the scheme uses a range-based method to locate ordinary nodes in the first phase. Then, it uses a range-free method, in the second phase, to locate ordinary nodes that were not located in the first phase. 
International Journal of Computer Networks \& Communications (IJCNC) Vol.12, No.3, May 2020

- Multi/single-stage: as the localization process progresses, more unknown nodes are able to determine their locations. Some of the localization schemes mark these nodes as reference nodes and allow them to participate in locating other nodes. Such schemes are called multi-stage schemes. These schemes can speed up the localization process and increase the localization coverage with a small number of special devices. However, localization errors accumulate in each step due to the possible errors that occur in the distance estimation. In contrast, the localization schemes that do not allow ordinary nodes to help locating other nodes are called single-stage localization schemes. The main drawback of these schemes is the high delay, and the need for more anchor nodes to achieve high coverage.

- Distributed/centralized: In distributed localization schemes, ordinary nodes estimate their location by themselves. In centralized schemes, on the other hand, location estimation is performed in a central station at the water surface, and nodes might not need to know their locations.

- Active/silent localization: In active localization schemes, ordinary nodes also exchange packets to complete the localization process. On the other hand, ordinary nodes in silent localization schemes passively listen to the localization packets transmitted by the anchor nodes.

\section{EXISTING LOCALIZATION SCHEMES}

This section reviews some of the existing localization schemes for UWSNs and classifies them based on range measurement. These schemes are classified based on the remaining categories described earlier in Table 2.

\subsection{Range-Based Schemes}

\section{- Dive and rise schemes}

M. Erol et al.[14], [6] and [15] proposed dive and rise localization schemes. The scheme in [14], called DNR, is based on mobile devices called DNR beacons that can dive and rise using a weight/bladder idea (i.e. dive with weight force, and rise using an air bladder). The scheme assumes that the DNR beacons and ordinary nodes are equipped with pressure sensors to determine their z-coordinate. The main idea of DNR is that each beacon device rises to the surface to obtain its location from GPS. Then, it dives underwater in a straight vertical path while broadcasting its location coordinates. Each ordinary node uses these localization packets for range measurements. Then, it applies either the bounding box or triangulation methods to estimate its location. Despite its simplicity, the diving and rising of the DNR beacons take longer time; thus, the localization speed and coverage depends mainly on the speed of the beacons and their numbers.

Due to the slow speed of the localization process in DNR, which is mainly caused by the slow speed of the DNR beacons, a Multi-stage Localization (MLS) Scheme is proposed in [6]. It incorporates an iterative procedure such that the located ordinary nodes act as reference nodes and can broadcast their location coordinates to help to locate other nodes. Three localization packets from three non-collinear sources are used to estimate the location of the node via lateration method. Although MLS improves the localization speed and deployment cost, this is achieved at the expense of high localization error and high communication overhead. 
In [15], an AUV-Aided Localization (AAL) is proposed in which one AUV device is used to locate sensor nodes. The AUV receives its position from GPS while floating to the sea surface. It then dives underwater to a certain depth and follows a certain path during which it can calculate its new positions. The main idea is that when the AUV patrols the network, it sends a wakeup message, and a node upon receiving this message sends a localization request. The AUV responds with a reply message that contains its coordinates. Using these request-reply messages, sensor nodes are able to calculate the round-trip time, which is then used along with the acoustic speed to estimate their distances to the AUV. By receiving three coordinates messages from three non-coplanar AUV locations, nodes use either the triangulation method or the bounding box method to estimate their xy-locations. The use of a single AUV, delays the localization process; hence, AAL might not be suitable for mobile networks where the localization is needed to be run periodically.

M. Beniwalet al.[16] proposed a localization scheme called Localization Scheme without Time Synchronization (LSWTS). LSWTS is an improvement over the DNR scheme of [14] by removing the requirement of time synchronization. The DNR beacons after receiving their location information from GPS dive vertically underwater and broadcast localization packets at fixed time intervals. An ordinary node passively listens to these packets, and when it captures two localization packets from a beacon device at two different times, it calculates its distance to that beacon. Then, if the node determines its distances to three beacons, it applies the trilateration method to estimate its location. Figure 6 demonstrates a scenario of the scheme. Although LSWTS locates nodes without time synchronization, its coverage depends on the number and distribution of the beacon nodes as it requires at least three beacon nodes to be in the range of each node to be localized.

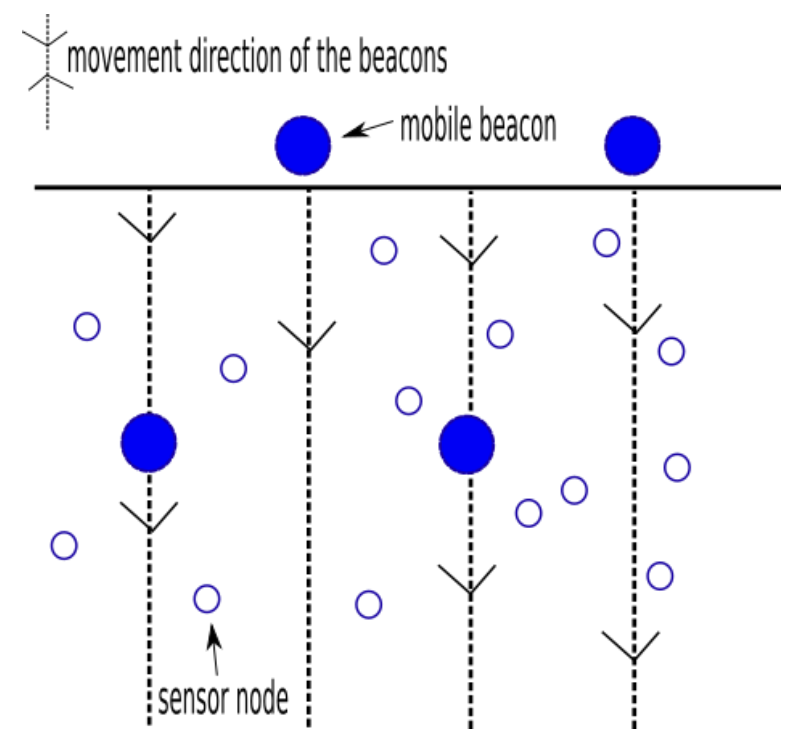

Figure 5: Illustration of the LSWTS scheme

\section{- $\quad$ Projection-based schemes}

M. Isiket al.[17] proposed a 3D Underwater Localization (3DUL) scheme. 3DUL assumes that there are three surface buoys with known locations acting as reference nodes. These surface buoys broadcast their location coordinates in the network. Ordinary nodes that receive three location packets from three different surface buoys determine their distances to these buoys via the two-way ranging method (i.e. request/reply packet exchange). Then, each ordinary node projects the locations of the buoys into its plane and uses the trilateration 
method to estimate itslocation. Located nodes become reference nodes and broadcast their locations. This iterative process continues until all nodes in the network are located. Since the ordinary nodes may move from their positions, those that have been labelled as reference nodes remain as a reference for a limited time according to their movement patterns. Although the two-way ranging method, as mentioned earlier, eliminates the need for clock synchronization, it might incur extra delay and overhead to locate the nodes. Furthermore, the iterative procedure increases the localization errors due to the error accumulation in the distance estimation.

A. Teymorianet al.[8] proposed a distributed localization scheme called Underwater Sensor Positioning (USP) for sparse 3D UWSNs. It is based on transforming the 3D localization problem into 2D via the projection technique. The protocol assumes that there are three surface buoys equipped with GPS receivers. Additionally, ordinary nodes are equipped with pressure sensors to learn their depth (z-coordinate). USP is an iterative localization scheme such that the localization procedure is executed in a predefined number of iterations. Each located node (including ordinary nodes) participates in the localization process by broadcasting locations of their own and their neighbours. Every non-located node upon receiving a localization packet, calculates its distance to the associated reference nodes. After that, it projects these reference nodes to its plane. If the projection is non-degenerative, the node estimates its location via the lateration method. The main issues in the USP scheme are: (i) surface buoys are selected randomly, which might affect localization coverage, (ii) it incurs communication overhead since each node should broadcast the location information it has about itself and its neighbours, and (iii) it suffers from cumulative localization errors as ordinary nodes with known locations act as reference nodes.

W. Cheng et al.[9] proposed a localization scheme called Localization Scheme for Large Scale UWSNs (LSLS). LSLS is based on a projection technique similar to USP. The scheme is suitable for large scale UWSNs. It consists of three phases as follows. In the first phase, the surface buoys broadcast their locations. Ordinary nodes that are in the range of these buoys estimate their locations via projection and trilateration. In the second phase, the protocol selects some of those located nodes, based on their distribution in the network, to act as reference nodes. Ordinary nodes that are still not located by the end of the second phase, broadcast localization requests. A new set of reference nodes are then selected to locate those nodes by broadcasting their locations as in the first two phases. Although LSLS is a multi-stage scheme, it reduces the communication overhead by only selecting a set of reference nodes which also increases the localization coverage. Though, the communication overhead is still high due to the control packets exchanged during ranging estimation.

M. Moradiet al.[13] proposed a centralized localization scheme called a Reverse Localization Scheme (RLS) for low communication overhead and fast localization. It assumes that there are surface buoys with known locations deployed at the water surface along with a sink node. The localization process is executed by the sink node on demand (i.e. when a sensor node detects an event to be reported to the sink). The main idea of RLS is summarized as follows. When an ordinary node detects an event, it broadcasts the detected event along with its ID, transmitting time and its depth (learnt from the pressure sensor). Each surface buoy upon receiving such a packet, injects its location coordinates and packet receiving time, and sends the packet to the sink. Then, the sink node is able to estimate the source node's location after receiving at least three packets from three surface buoys via projection (i.e. sink node projects the source node to the surface level) and trilateration. RLS differs from the existing schemes by locating only those nodes that detect events. Other nodes remain non-located, which might be cost and time effective. Nevertheless, ordinary nodes remain unaware of their locations; hence, the scheme is not suitable for location-based routing. 
Z. Zhou et al.[18] proposed a hierarchical Localization Scheme for Large scale networks (LSL). The authors assume that there are at least 4 surface buoys with GPS-receivers and anchor nodes scattered in the 3D network. Additionally, they assume that the anchor nodes are in the communication range of the surface buoys and they are localized with their help via some of the existing techniques. All anchor nodes are assumed to be reference nodes (i.e. nodes with known locations) with a high confidence value. LSL can be summarized as follows. The reference nodes periodically broadcast localizationpackets containing their location coordinates. Ordinary nodes estimate their distances to the one-hop reference nodes using ToA. To speed up the localization process and increase its coverage, they also estimate the non-neighboring distances using the 3D Euclidean distance estimation method. Then, if the node estimates its distance to 4 non-collinear reference nodes, it calculates its location and the confidence value. If the confidence value is above a predefined threshold, it labels itself as a reference node and starts broadcasting its location. Although the recursive procedure of the scheme makes it suitable for large-scale networks, it is not suitable for mobile networks due to the number of control packets that need to be exchanged in the localization process.

A Scalable Localization with Mobility Prediction (SLMP) [19] scheme is proposed for large scale mobile UWSNs. The authors utilized underwater mobility characteristics and spatial correlation in the movement of underwater objects (i.e. group movement) to construct a mobility prediction algorithm. This algorithm is then used in the localization process. Basically, the localization in the SLMP consists of two phases, namely; anchor nodes localization and ordinary nodes localization. As in LSL, surface buoys are used to localize anchor nodes. In the second phase, every anchor node runs the mobility prediction algorithm to determine its mobility pattern, and then broadcasts it along with its location coordinates as a localization message. Ordinary nodes utilize such localization messages to determine their locations and mobility patterns. Particularly, each ordinary node records the information received in the localization messages. It runs its mobility prediction algorithm to determine its mobility pattern, which is then used to estimate its location. Since the mobility pattern may become invalid by time, an anchor node calculates its new location periodically and compares it with the predicted location using the mobility pattern. If the Euclidean distance between these locations is below a predefined threshold, then the mobility pattern is still valid, and the anchor node remains silent for the next period. Otherwise, if the distance is above the threshold, it runs the mobility prediction algorithm to obtain a new mobility pattern and broadcasts a new localization message. The mobility pattern of the ordinary node is assumed to be valid until it receives a new localization message. The performance of the scheme depends on the mobility pattern. In particular, less overhead and less energy consumption are achieved when the changes in the mobility pattern is minimized and vice versa.

H. Huang et al.[20] proposed node localization scheme (NL-AoA) with AoA assistant in multi-hop networks. NL-AoA is proposed for large-scale sparse UWSNs. It assumes a static network and that sensor nodes are capable of measuring AoA of signals. The scheme consists of two phases; distance estimation and location estimation. In the first phase, distance is estimated using AoA method. Since in sparse networks, a sensor node might not be in a communication range with a sufficient number of anchor nodes, the authors propose a new distance estimation method by estimating rotation matrix for nodes that are nonneighbors to anchor nodes. When a sensor node estimates the distances to four anchor nodes, it applies weighted least square method to estimate its location. 
A localization scheme called Anchor-Free Localization Algorithm (AFLA) [21] is proposed for low cost and high flexibility. AFLA does not require special nodes such as anchors, surface buoys or AUVs to localize ordinary nodes. Instead, it is a self-localization scheme that uses the relationship between adjacent nodes to localize them. It assumes that each node can have at least two neighbouring nodes. In addition, it assumes that sensor nodes are attached to cables (of known length) anchored to the bottom surface. These anchors limit the movement of the attached sensor nodes in limited areas as illustrated by the dashed ovals in Figure 7. Furthermore, each node is equipped with pressure (depth) sensor to determine its depth; hence, reduces the 3D localization problem to a $2 \mathrm{D}$. Therefore, each node can determine its spherical centre's coordinates, depth and cable length at any point of time. Nodes calculate their locations by exchanging their spherical centre's coordinates, depth and cable length with their neighbours. This information is used to calculate the distances to neighbouring nodes, which are then used to estimate location coordinates.

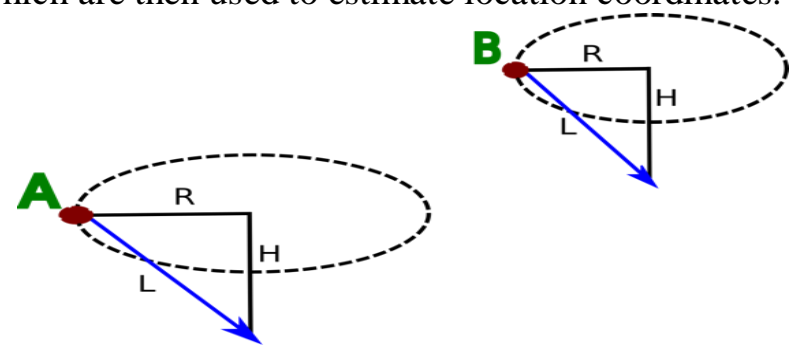

Figure 6: Illustration of the limited areas in AFLA

\section{- $\quad$ Schemes based on distance differences}

Z. Qianget al.[22] proposed a synchronization-free localization scheme called Clock Synchronization Independent Localization Scheme (CSILS). The scheme assumes that there are four surface buoys with GPS receivers used to locate the underwater ordinary nodes. One of these buoys is assumed to be the master and, it is responsible for estimating the locations of the ordinary nodes. Instead of estimating distances between surface buoys and ordinary nodes, CSILS estimate distance differences based on local clocks. The localization process is performed on demand such that when an ordinary node senses an event, it broadcasts a packet to the surface. The other three buoys upon receiving such a packet send a reply packet to the master. The master buoy then estimates the location of the ordinary node based on distance differences. It is worth mentioning that the performance of the scheme is highly depending on the location of the anchor nodes as shown in the simulation results. Moreover, the scheme requires that the four anchor nodes should be in the communication range of the ordinary nodes, which means that ordinary nodes should havea sufficient transmission range. In addition, CSILS is unable to uniquely estimate the location of an ordinary node with less than four anchors.

\subsection{Range-Free Schemes}

V. Chandrasekhar et al.[23] proposed an Area-based Localization Scheme (ALS). Instead of providing an exact location of the nodes, the ALS estimates the nodes' location within a certain region. It works as follows. Each surface buoy sends beacon packets at different power levels and at different time intervals. Sensor nodes upon receiving these beacons record the lowest power level received from each anchor nodes. Then, they forward this information to the sink nodes at the surface. Sink nodes use this information to estimate the region at which each sensor is 
International Journal of Computer Networks \& Communications (IJCNC) Vol.12, No.3, May 2020

located. The coverage and granularity of the ALS are limited by the number of anchor nodes and their positions, and the number of transmitted power levels and their values. Furthermore, the scheme does not consider the mobility of the nodes and it assumes $2 \mathrm{D}$ environment.

In [24], the authors proposed a 3D Multi power Area Localization Scheme (3D-MALS) based on mobile Detacheable Elevator Tranceivers (DETs). It is an extension of ALS by adding the third dimesnion (i.e. depth). Nodes obtain their depth information via pressure sensors. In addition to devices used in ALS, 3D-MALS uses DETs, which are attched to the surface buoys to get location cooridnates. Then, they dive vertically underwater and broadcast their location information at different power levels. Ordinary nodes record a list of these information along with their corresponding lowest transmitted power levels. Then, they send them to the sink nodes to estimate the region at which each ordinary node is located. The sink nodes use the ALS algorithm to estimate the location.

\subsection{Hybrid Localization Schemes}

J. Luo et al.[25] proposed a localization scheme called Two-Phase Time Synchronization-Free Localization Algorithm (TP-TSFLA). The scheme assumes a static network and nodes are equipped with pressure sensors to obtain their depths. The protocol consists of two phases. In the first phase, a number of mobile beacon nodes with known locations dive and rise in a vertical direction, and broadcast localization packets in fixed time intervals similar to LSWTS [16]. Each ordinary node upon receiving such packet, applies the same method as in [16] to calculate the distance to the beacon node. Then, if the ordinary node successfully computes the distances to at least three beacons, it uses the Particle Swarm Optimization (PSO) [26][27] to estimate its location. Since the number of ordinary nodes is much higher than the number of beacon nodes, some of the ordinary nodes will not be able to receive packets from at least three different beacon nodes. Therefore, in the second phase, the nodes that were unable to receive enough packets, broadcast localization requests. Ordinary nodes that were localized in the first phase will act as reference nodes and reply to those requests by sending their coordinates. Upon receiving such packets, the still non-located nodes apply the Circle-based Range-Free Localization Algorithm (CRFLA) proposed in the paper to determine their locations. To improve the accuracy of the estimated locations in the second phase, the authors proposed an extension of CRFLA that helps adjusting the estimated locations.

Table 1: Comparison between existing localization schemes for UWSNs

\begin{tabular}{|c|c|c|c|c|c|c|c|c|c|}
\hline \multicolumn{4}{|c|}{$\begin{array}{l}\text { Localization } \\
\text { Scheme }\end{array}$} & \multirow{2}{*}{\begin{tabular}{c} 
Centralized \\
\multicolumn{1}{l}{} \\
Distributed \\
Distributed
\end{tabular}} & \multirow{2}{*}{ 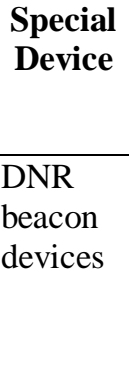 } & \multirow{2}{*}{$\begin{array}{c}\begin{array}{c}\text { Synchroni } \\
\text { zation } \\
\text { Required }\end{array} \\
\checkmark\end{array}$} & \multirow{2}{*}{$\begin{array}{c}\begin{array}{c}\text { Nodes } \\
\text { Mobility }\end{array} \\
\checkmark\end{array}$} & \multirow{2}{*}{$\begin{array}{l}\begin{array}{c}\text { Dimen } \\
\text { sion }\end{array} \\
2 \mathrm{D}\end{array}$} & \multirow{2}{*}{\begin{tabular}{l}
\multicolumn{1}{c}{ Remarks } \\
-Simple \\
implementatio \\
$\mathrm{n}$
\end{tabular}} \\
\hline  & 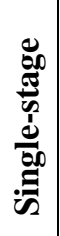 & 莺 & 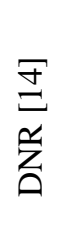 & & & & & & \\
\hline
\end{tabular}


International Journal of Computer Networks \& Communications (IJCNC) Vol.12, No.3, May 2020

\begin{tabular}{|c|c|c|c|c|c|c|c|c|}
\hline & & 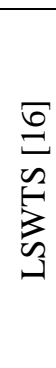 & Distributed & $\begin{array}{l}\text { DNR } \\
\text { beacon } \\
\text { devices }\end{array}$ & $x$ & $x$ & $2 \mathrm{D}$ & $\begin{array}{l}\text {-Enhancement } \\
\text { over DNR } \\
\text { scheme [14] } \\
\text {-Eliminates } \\
\text { the need for } \\
\text { time } \\
\text { synchronizatio } \\
\text { n with less } \\
\text { overhead }\end{array}$ \\
\hline & & $\frac{\sqrt[n]{己}}{\frac{1}{2}}$ & Distributed & $1 \mathrm{AUV}$ & $x$ & $\checkmark$ & $2 \mathrm{D}$ & $\begin{array}{l}\text {-Cost effective } \\
\text { in terms of } \\
\text { special } \\
\text { devices but } \\
\text { high delay }\end{array}$ \\
\hline & : & $\begin{array}{l}\frac{\sigma}{2} \\
\underline{n} \\
\underline{a}\end{array}$ & Centralized & $\begin{array}{l}\text {-Surface } \\
\text { buoys } \\
\text { - Sink } \\
\text { node }\end{array}$ & $\checkmark$ & $\checkmark$ & $2 \mathrm{D}$ & $\begin{array}{l}\text {-The sink node } \\
\text { is responsible } \\
\text { for location } \\
\text { calculation } \\
\text {-Ordinary } \\
\text { nodes need not } \\
\text { to know their } \\
\text { locations } \\
\text {-Not suitable } \\
\text { for location- } \\
\text { based routing } \\
\text { protocols }\end{array}$ \\
\hline & & 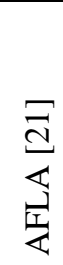 & Distributed & $\begin{array}{l}\text { No } \\
\text { special } \\
\text { device }\end{array}$ & $\checkmark$ & $\checkmark$ & $2 \mathrm{D}$ & $\begin{array}{lr}\text {-Does not } \\
\text { require any } \\
\text { special device }\end{array}$ \\
\hline & & 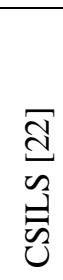 & Centralized & $\begin{array}{l}\text { Surface } \\
\text { buoys }\end{array}$ & $x$ & $\checkmark$ & $2 \mathrm{D}$ & $\begin{array}{l}\text {-Calculates } \\
\text { distance } \\
\text { difference } \\
\text { instead of } \\
\text { distances to } \\
\text { anchor nodes }\end{array}$ \\
\hline 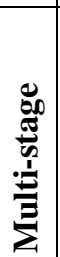 & $\frac{\overrightarrow{0}}{\bar{v}}$ & $\frac{\sigma}{\frac{\sigma}{n}}$ & Distributed & $\begin{array}{l}\text { DNR } \\
\text { beacon } \\
\text { devices }\end{array}$ & $\checkmark$ & $\checkmark$ & $2 \mathrm{D}$ & $\begin{array}{l}\text {-Enhancement } \\
\text { over DNR } \\
\text { scheme [14] }\end{array}$ \\
\hline
\end{tabular}


International Journal of Computer Networks \& Communications (IJCNC) Vol.12, No.3, May 2020

\begin{tabular}{|c|c|c|c|c|c|c|c|}
\hline & $\begin{array}{l}\stackrel{\sigma}{\Xi} \\
\sum_{\bar{\sigma}}\end{array}$ & Distributed & $\begin{array}{l}\text { Surface } \\
\text { buoys } \\
\text { - Anchor } \\
\text { nodes }\end{array}$ & $\begin{array}{l}\text { Not } \\
\text { specified }\end{array}$ & $\checkmark$ & $3 \mathrm{D}$ & $\begin{array}{l}\text {-Uses } \\
\text { prediction of } \\
\text { node mobility } \\
\text { pattern } \\
\text { - Localization } \\
\text { is based on the } \\
\text { developed } \\
\text { mobility } \\
\text { prediction } \\
\text { algorithm }\end{array}$ \\
\hline \multirow{5}{*}{ 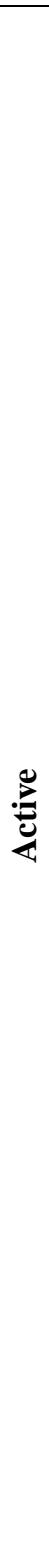 } & 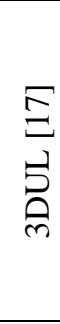 & Distributed & $\begin{array}{l}3 \\
\text { surface } \\
\text { buoys }\end{array}$ & $x$ & $\checkmark$ & $2 \mathrm{D}$ & $\begin{array}{l}\text {-Cost effective } \\
\text { in terms of } \\
\text { special } \\
\text { devices } \\
\text {-Possibility of } \\
\text { high } \\
\text { localization } \\
\text { error }\end{array}$ \\
\hline & $\begin{array}{l}\infty \\
\infty \\
\tilde{\omega}\end{array}$ & Distributed & $\begin{array}{l}3 \\
\text { surface } \\
\text { buoys }\end{array}$ & $\begin{array}{l}\text { Not } \\
\text { specified }\end{array}$ & $x$ & $2 \mathrm{D}$ & $\begin{array}{l}\text {-Designed } \\
\text { mainly for } \\
\text { sparse 3D } \\
\text { UWSNs } \\
\text {-High } \\
\text { communicatio } \\
\text { n overhead }\end{array}$ \\
\hline & $\begin{array}{l}\sigma \\
\sqrt{3} \\
\text { n }\end{array}$ & Distributed & $\begin{array}{l}3 \\
\text { surface } \\
\text { buoys }\end{array}$ & $x$ & $x$ & $2 \mathrm{D}$ & $\begin{array}{lr}\text {-Uses } & \text { a } \\
\text { technique } & \text { to } \\
\text { select } & \\
\text { reference } & \\
\text { nodes } & \end{array}$ \\
\hline & $\begin{array}{l}\infty \\
= \\
= \\
=\end{array}$ & Distributed & $\begin{array}{l}-4 \\
\text { surface } \\
\text { buoys } \\
\text {-Anchor } \\
\text { nodes }\end{array}$ & $\checkmark$ & $\checkmark$ & $3 \mathrm{D}$ & $\begin{array}{l}\text {-Expands the } \\
\text { 2D Euclidean } \\
\text { distance } \\
\text { estimation to a } \\
\text { 3D }\end{array}$ \\
\hline & 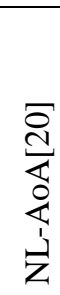 & Distributed & $\begin{array}{l}\text { Anchor } \\
\text { nodes }\end{array}$ & $\checkmark$ & $x$ & $\begin{array}{ll}2 \mathrm{D} & \& \\
3 \mathrm{D} & \end{array}$ & $\begin{array}{l}\text { Utilizes multi- } \\
\text { hop } \\
\text { communicatio } \\
\text { ns to localize } \\
\text { sensor nodes }\end{array}$ \\
\hline
\end{tabular}


International Journal of Computer Networks \& Communications (IJCNC) Vol.12, No.3, May 2020

\begin{tabular}{|c|c|c|c|c|c|c|c|c|c|}
\hline \multirow[t]{2}{*}{ 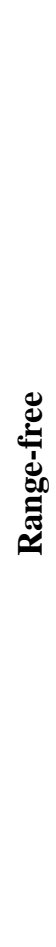 } & 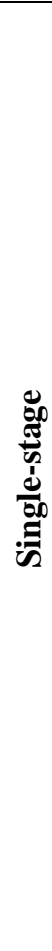 & \multirow[t]{2}{*}{$\sum_{\bar{Z}}^{\grave{Z}}$} & $\begin{array}{l}\bar{d} \\
\frac{d}{2} \\
\frac{1}{4}\end{array}$ & Centralized & $\begin{array}{l}\text {-Sink } \\
\text { nodes } \\
\text {-Surface } \\
\text { buoys }\end{array}$ & $x$ & $x$ & $2 \mathrm{D}$ & $\begin{array}{l}\text { Complex } \\
\text { calculations } \\
\text { are performed } \\
\text { by the sink } \\
\text { nodes rather } \\
\text { than ordinary } \\
\text { nodes. } \\
\text { Designed for } \\
\text { 2D } \\
\text { environment }\end{array}$ \\
\hline & & & $\sum_{\substack{1 \\
\infty}}^{\infty}$ & Centralized & $\begin{array}{l}\text {-DETs } \\
\text {-Surface } \\
\text { buoys } \\
\text {-Sink } \\
\text { nodes }\end{array}$ & $x$ & $x$ & $2 \mathrm{D}$ & $\begin{array}{l}\text { A 3D version } \\
\text { of ALS } \\
\text { scheme }\end{array}$ \\
\hline 党 & & & 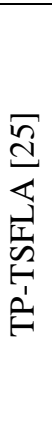 & Distributed & $\begin{array}{l}\text { - Beacon } \\
\text { nodes }\end{array}$ & $x$ & $x$ & $2 \mathrm{D}$ & 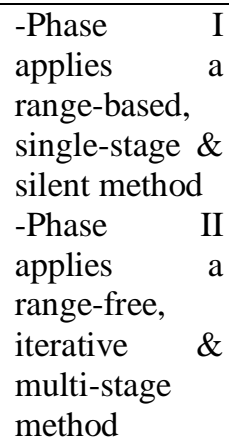 \\
\hline
\end{tabular}

\section{OPEN RESEARCH ISSUES}

Despite the research efforts in developing localization schemes, there remains a number of issues that pose open research questions. In this section we list major problems in the area that still require further investigation.

- Geographic routing protocols are built on top of some localization schemes. Such routing protocols assume that all nodes can obtain their locations accurately. In reality, however, some nodes might not be able to obtain their locations and some others obtain inaccurate locations. One possible reason is that a node might not be able to receive a sufficient number of messages required for location estimation. Another reason is related to the errors in distance estimation. Several studies have shown that localization inaccuracies degrade the performance of location-based routing protocols in WSNs [28][29][30][31]. To the best of our knowledge, however, no work studied the impact of localization schemes on the performance of geographic routing in UWSNs. Studying the impacts of localization schemes on the performance of geographic routing protocols is still an open research issue.

- The developed localization schemes are analyzed assuming constant sound speed. However, the speed of sound underwater is affected by different conditions such as 
International Journal of Computer Networks \& Communications (IJCNC) Vol.12, No.3, May 2020

temperature, salinity and depth. It increases by $4.0 \mathrm{~m} / \mathrm{s}$ when the temperature increases by $1^{\circ} \mathrm{C}$ [32]. When the salinity of the water increases by 1 practical salinity unit (PSU), the speed increases by $1.4 \mathrm{~m} / \mathrm{s}$ [32]. By increasing the depth under water by $1 \mathrm{~km}$, the speed of sound increases by $17 \mathrm{~m} / \mathrm{s}$ [32]. The assumption of constant sound speed may increase the error in the estimation of the sensor nodes' locations. Therefore, developing a model that reflects the change in the speed of sound underwater and incorporating this in the location estimation of the nodes is still an open question.

- Underwater nodes move due to different reasons and hence there is a need for developing a more realistic mobility model that reflects the mobility of the sensor nodes in different operating conditions and environments. Then, the next step is to analyze the performance of the localization schemes using this mobility model.

\section{CONCLuSiON}

Localization in UWSNs is one of the critical tasks in designing UWSNs. It can be used for data tagging, object tracking and data transmission in geographic routing protocols. Several factors and challenges need to be considered when developing a localization scheme for UWSNs. Characteristics of the acoustic signal, nodes' deployment, node mobility, and time synchronization are examples of these challenges. This paper has reviewed the state-of-the-art of the localization schemes in UWSNs. It has surveyed some of the recent schemes and has classified them in different categories. Furthermore, the paper has discussed some open research issues that need further investigation in this area.

\section{ACKNOWLEDGMENT}

This work is supported by The Research Council (TRC) of the Sultanate of Oman under the research grant number RC/SCI/COMP/15/02.

\section{REFERENCES}

[1] M. Erol-Kantarci, H. T. Mouftah, and S. Oktug, "A Survey of Architectures and Localization Techniques for Underwater Acoustic Sensor Networks," IEEE Communications Surveys and Tutorials, vol. 13, no. 3, pp. 487-502, September 2011.

[2] M. Beniwal and R. Singh, "Localization Techniques and Their Challenges in Underwater Wireless Sensor Networks," International Journal of Computer Science and Information Technologies (IJCSIT), vol. 5, no. 3, pp.4706-4710, 2014.

[3] H.-P. Tan, R. Diamant, W. K. G. Seah, and M. Waldmeyer, "A survey of techniques and challenges in underwater localization," Ocean Engineering, vol. 38, no. 14, pp. 1663-1676, October 2011.

[4] G. Han, C. Zhang, L. Shu, and J. J. P. C. Rodrigues, "Impacts of Deployment Strategies on Localization Performance in Underwater Acoustic Sensor Networks," IEEE Transactions on Industrial Electronics, vol. 62, no. 3, pp. 1725-1733, March 2015.

[5] M. Erol-Kantarci, S. Oktug, L. Vieira, and M. Gerla, "Performance evaluation of distributed localization techniques for mobile underwater acoustic sensor networks," Ad Hoc Networks, vol. 9, no. 1, pp. 61-72, January 2011.

[6] M. Erol, L. F. M. Vieira, A. Caruso, F. Paparella, M. Gerla, and S. Oktug, "Multi Stage Underwater Sensor Localization Using Mobile Beacons," in 2008 Second International Conference on Sensor Technologies and Applications (sensorcomm 2008), 25-31 August 2008, pp. 710-714, Cap Esterel, France.

[7] A. Koubâa and M. Ben Jamâa, "Taxonomy of Fundamental Concepts of Localization in CyberPhysical and Sensor Networks," Wireless Personal Communications, vol. 72, no. 1, pp. 461-507, September 2013.

[8] A. Y. Teymorian, W. Cheng, L. Ma, X. Cheng, X. Lu, and Z. Lu, "3D Underwater Sensor Network Localization," IEEE Transactions on Mobile Computing, vol. 8, no. 12, pp. 1610-1621, December 2009. 
International Journal of Computer Networks \& Communications (IJCNC) Vol.12, No.3, May 2020

[9] W. Cheng, A. Thaeler, X. Cheng, F. Liu, X. Lu, and Z. Lu, "Time-Synchronization Free Localization in Large Scale Underwater Acoustic Sensor Networks," in 2009 29th IEEE International Conference on Distributed Computing Systems Workshops, 22-26 June 2009, pp. 80-87, Montreal, QC, Canada.

[10] A. Mesmoudi, M. Feham, and N. Labraoui, "Wireless sensor networks localization algorithms: a comprehensive survey," arXiv preprint arXiv:1312.4082, December 2013.

[11] D. Niculescu and B. Nath, "DV Based Positioning in Ad Hoc Networks," Telecommunication Systems, vol. 22, no. 1-4, pp. 267-280, January 2003.

[12] T. He, C. Huang, B. M. Blum, J. A. Stankovic, and T. Abdelzaher, "Range-free localization schemes for large scale sensor networks," in Proceedings of the 9th annual international conference on Mobile computing and networking - MobiCom '03, 14-19 September 2003, pp. 81-95, San Diego, CA, USA.

[13] M. Moradi, J. Rezazadeh, and A. S. Ismail, "A Reverse Localization Scheme for Underwater Acoustic Sensor Networks," Sensors, vol. 12, no. 12, pp. 4352-4380, March 2012.

[14] M. Erol, L. F. M. Vieira, and M. Gerla, "Localization with Dive'N'Rise (DNR) beacons for underwater acoustic sensor networks," in Proceedings of the second workshop on Underwater networks - WuWNet '07, 14 September 2007, pp. 97-100, Montreal, Quebec, Canada.

[15] M. Erol, L. F. M. Vieira, and M. Gerla, "AUV-Aided Localization for Underwater Sensor Networks," in International Conference on Wireless Algorithms, Systems and Applications (WASA 2007), 1-3 August 2007, pp. 44-54, Chicago, IL, USA.

[16] M. Beniwal, R. P. Singh, and A. Sangwan, "A Localization Scheme for Underwater Sensor Networks Without Time Synchronization," Wireless Personal Communications, vol. 88, no. 3, pp. 537-552, June 2016.

[17] M. Isik and O. Akan, "A three dimensional localization algorithm for underwater acoustic sensor networks," IEEE Transactions on Wireless Communications, vol. 8, no. 9, pp. 4457-4463, September 2009.

[18] Z. Zhou, J.-H. Cui, and S. Zhou, "Efficient localization for large-scale underwater sensor networks," Ad Hoc Networks, vol. 8, no. 3, pp. 267-279, May 2010.

[19] Z. Zhou, Z. Peng, J.-H. Cui, Z. Shi, and A. Bagtzoglou, "Scalable Localization with Mobility Prediction for Underwater Sensor Networks," IEEE Transactions on Mobile Computing, vol. 10, no. 3, pp. 335-348, March 2011.

[20] H. Huang and Y. R. Zheng, "Node localization with AoA assistance in multi-hop underwater sensor networks," Ad Hoc Networks, vol. 78, pp. 32-41, September 2018.

[21] Y. Guo and Y. Liu, "Localization for anchor-free underwater sensor networks," Computers \& Electrical Engineering, vol. 39, no. 6, pp. 1812-1821, August 2013.

[22] Z. Qiang, Z. Senlin, and L. Meiqin, "A clock synchronization independent localization scheme for underwater wireless sensor networks," in Proceedings of the Eighth ACM International Conference on Underwater Networks and Systems - WUWNet '13, 11-13 November 2013, pp. 1-5, Kaohsiung, Taiwan.

[23] V. Chandrasekhar and W. Seah, "An Area Localization Scheme for Underwater Sensor Networks," in OCEANS 2006 - Asia Pacific, 16-19 May 2006, pp. 1-8, Singapore, Singapore.

[24] Y. Zhou, B. Gu, K. Chen, J. Chen, and H. Guan, "An range-free localization scheme for large scale underwater wireless sensor networks," Journal of Shanghai Jiaotong University (Science), vol. 14, no. 5, pp. 562-568, October 2009.

[25] J. Luo and L. Fan, "A Two-Phase Time Synchronization-Free Localization Algorithm for Underwater Sensor Networks," Sensors, vol. 17, no. 12, p. 726, March 2017.

[26] R. Poli, J. Kennedy, and T. Blackwell, "Particle swarm optimization," Swarm Intell., vol. 1, no. 1, pp. 33-57, October 2007.

[27] Jialiang Lv, Huanqing Cui, and Ming Yang, "Distribute localization for wireless sensor networks using particle swarm optimization," in 2012 IEEE International Conference on Computer Science and Automation Engineering, 22-24 June 2012, pp. 355-358, Beijing, China.

[28] B. Peng and A. H. Kemp, "Energy-efficient geographic routing in the presence of localization errors," Computer Networks, vol. 55, no. 3, pp. 856-872, February 2011.

[29] M. Kadi and I. Alkhayat, "The effect of location errors on location based routing protocols in wireless sensor networks," Egyptian Informatics Journal, vol. 16, no. 1, pp. 113-119, March 2015. 
International Journal of Computer Networks \& Communications (IJCNC) Vol.12, No.3, May 2020

[30] R. C. Shah, A. Wolisz, and J. M. Rabaey, "On the performance of geographical routing in the presence of localization errors," in IEEE International Conference on Communications, 2005. ICC 2005, 16-20 May 2005, vol. 5, pp. 2979-2985, Seoul, South Korea.

[31] D. Son, A. Helmy, and B. Krishnamachari, "The effect of mobility-induced location errors on geographic routing in mobile ad hoc sensor networks: analysis and improvement using mobility prediction," IEEE Transactions on Mobile Computing, vol. 3, no. 3, pp. 233-245, July 2004.

[32] M. Ayaz, I. Baig, A. Abdullah, and I. Faye, "A survey on routing techniques in underwater wireless sensor networks," Journal of Network and Computer Applications, vol. 34, no. 6, pp. 1908-1927, November 2011.

\section{AUTHORS}

Dr. Faiza Al-Salti received her B.Sc., M.Sc. and Ph.D. Degrees in computer science from the Sultan Qaboos University (Oman) in 2012, 2015 and 2019, respectively. She is currently an assistant professor in Muscat College (Oman). Her research interest includes communication protocols, terrestrial and underwater wireless sensor networks, and the Internet of Things.

Dr. Nasser Alzeidi received his Ph.D. degree in Computer Science from the University of Glasgow (UK) in 2007. He is currently an assistant professor of computer science and the director of the Center for Information Systems at Sultan Qaboos University, Oman. His research interests include performance evaluation of communication systems, wireless networks, interconnection networks, System on Chip architectures and parallel and distributed computing. He is a member of the IEEE.

Prof. Khaled Day received his undergraduate degree in computer science from the University of Tunis in 1986. He was awarded in 1986 the 'President Habib Bourguiba Prize' for excellent academic performance upon completion of his undergraduate degree. He was then awarded from the Tunisian government a scholarship to pursue graduate studies in the USA starting 1987. He obtained an M.Sc. and Ph.D. degrees in computer science from the University of Minnesota (USA) in 1989 and 1992 respectively. Dr. Day has worked at the University of

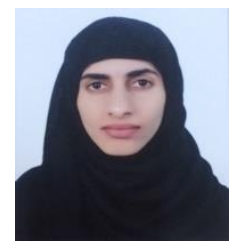
Bahrain in the period 1992-1996 as Assistant Professor. He then joined in 1996 Sultan Qaboos University as Assistant Professor. He was promoted to Associate Professor in 1999 and then to Professor in 2005. He served as the Head of the Department of Computer Science at Sultan Qaboos University during the periods 2000-2002 and 2006-2010. He was appointed in January 2013 as the Dean of Research of Sultan Qaboos University. His areas of research interest include interconnection networks, parallel algorithms, distributed systems, and wireless networks. He has published over 100 research papers in international journals and conferences. He has received in 2000 the Abdul Hameed Shoman Prize for Young Arab Researchers. He is a senior member of IEEE. 